\title{
ENCARTE DIGITAL
}

\section{Desafíos a la historia de América Latina}

\author{
Una visión de género
}

\author{
Challenges to Latin American history \\ A gender perspective
}

Sara Beatriz Guardia*

\begin{abstract}
Resumen: En América Latina la historiografía enfrenta dos retos insoslayables: la deconstrucción de una historia eurocéntrica basada en principios y valores considerados universales que distorsionaron la visión y estudio de las culturas que se desarrollaron a lo largo de varios miles de años antes de la conquista española y portuguesa, en el caso de Brasil; y la deconstrucción de una historia patriarcal que no visibiliza a las mujeres en los diferentes procesos de nuestra historia. Revertir y transformar esta situación es el objetivo de nuestro trabajo centrado en visibilizar a las mujeres en sus espacios, prácticas culturales, y participación en la historia del continente. Solo así tendremos una historia integral que recoja ambas experiencias.
\end{abstract}

Palabras claves: Deconstrucción. Historia. Eurocentrismo. Patriarcalismo.

\begin{abstract}
Latin America historiography faces two unavoidable challenges: The deconstruction of a Eurocentric history based on principles and values considered to be universal that distorted vision and study of cultures that developed over several thousands of years before the Spanish Conquest and Portuguese, in the case of Brazil; and the deconstruction of patriarchal story that does not make visible women in the different processes in our history. Reverse and transform this situation is the objective of our work focused on the visibility of women in their spaces, cultural practices, and participation in the history of the continent. Only then we will have a comprehensive history that collect both experiences.
\end{abstract}

Keywords: Deconstruction. History. Eurocentrism. Patriarchy.

\footnotetext{
* Escritora, profesora y investigadora de la Facultad de Ciencias de la Comunicación de la Universidad de San Martín de Porres, em Lima, no Peru<sarabeatriz@telefonica.net.pe>.
} 


\section{Antecedentes}

Hasta comienzos del siglo 21 las mujeres que figuran en el discurso histórico son excepcionales por su belleza, virtudes o heroísmo (Perrot, 1995, p. 71). Todas las demás no existen en una historia fundada en personajes de la élite, batallas y tratados políticos; una historia que registra e interpreta los distintos procesos y experiencias que ha vivido la humanidad a través de la visión, pensamientos y manifestaciones de quienes la han escrito. Todos hombres en su mayoría de clases y pueblos dominantes que se erigieron según el modelo androcéntrico, en el centro arquetípico del poder ejercido en el espacio público y en un tiempo cronológico (Moreno Sardá, 1986), de acuerdo a la división de lo privado y lo público que articula estructuralmente las sociedades jerarquizadas. Según lo cual los hombres aparecen como los únicos capaces de gobernar y dictar leyes, mientras las mujeres ocupan un lugar secundario, en el espacio privado y alejadas de los grandes acontecimientos de la historia.

El cambio en la historia oficial se produjo en el siglo 18 cuando el ámbito privado comenzó a configurarse separado del poder político (Nogal Fernández, 2006, p. 36). Hecho que constituye un punto de partida de la visibilidad de las mujeres, puesto que una historia que solo enfoca la esfera pública, entendida como el espacio de las relaciones de poder político y económico, significa una mirada de los hombres hacia los hombres. Aquí, las huellas públicas y privadas de las mujeres han quedado borradas, silenciadas en los archivos públicos, invisibles para la historia (Perrot, 1999, p. 13). Varios factores posibilitaron esta transformación: la Ilustración en la que razón y educación constituyeron características por excelencia; el liberalismo que planteó la igualdad aunque sin poder concretar su propuesta durante la revolución francesa cuando las mujeres demandaron que la Declaración de los derechos del hombre y del ciudadano las incluyera. El principio de que la igualdad, la libertad y la autonomía son comunes a todos los seres humanos, permitió que las mujeres articularan un proyecto como movimiento social con diferentes corrientes teóricas y tendencias que explican las causas de la subordinación de las mujeres y las estrategias del cambio.

Pero si las huellas de las mujeres han sido borradas, ¿Cómo podemos conocer sus formas de vivir la cotidianidad, e interpretar sus pensamientos, emociones y acciones? ¿Cómo aproximarnos a los hechos que originaron cambios desde las mujeres? ¿Qué sabemos de ellas si hasta los tenues rastros "provienen de la mirada de los hombres que gobiernan la ciudad, construyen su memoria y administran sus archivos"? (Duby-Perrot, 1991, p. 44). En 
definitiva, ¿cómo "dirigirse al sujeto históricamente mudo de la mujer", y ¿de qué manera conocer "el testimonio de la propia voz de la conciencia femenina”? (Spivak, 2011, p. 80). La única respuesta está en la deconstrucción de la historia con la inclusión de las mujeres como sujetos históricos; de lo contrario como bien dice Michelle Perrot, significaría llenar un "casillero" hasta ahora vacío de presencia femenina.

El inicio se situó en 1929, cuando coincidiendo con la crisis del capitalismo, Marc Bloch ${ }^{1}$ y Lucien Febvre fundaron en París la revista "Annales d'histoire économique et sociale", que transformó el concepto de la historia al priorizar una historia social que incluía mentalidades, vida cotidiana, costumbres, familia, sentimientos, y subjetividades colectivas, permitiendo así estudiar a las mujeres como sujetos históricos. Hasta entonces, se había ubicado a la familia en la esfera privada separada de otro tipo de relaciones sociales, lo que contribuyó a perpetuar una ideología de la domesticidad, y promover la invisibilidad de las mujeres como trabajadoras (Scott, 1992, p. 54). Tal como señala Lucien Febvre, la historia "no se hace en absoluto dentro de una torre de marfil. Se hace en la misma vida, y por seres vivos que están inmersos en el siglo" (Chartier, 2008, p. 16).

Coincidiendo con la aparición de la Escuela de los anales, un grupo de historiadoras inglesas fundaron la Conferencia de mujeres historiadoras de Berkshire, buscando influir en la American historical association dominada por los hombres. Pero más allá de la necesidad de permear las instituciones lo que se planteó entonces como aspecto primordial fue estudiar el pasado de las mujeres a través de los ojos de las mujeres. Data de 1933 el planteamiento pionero de la historiadora norteamericana, Mary Ritter Beard, con su libro, America through women's eyes (América a través de los ojos de las mujeres). ¿Qué idea tenían las mujeres de sí mismas? ¿Cómo percibían su presencia en la sociedad? ¿Cómo eran vistas por los hombres? Son algunas de las interrogantes que esta obra intentó responder, utilizando como fuentes los diarios, las novelas y la correspondencia personal.

En 1945, el historiador inglés William L. Schurz, incluyó un capítulo dedicado a las mujeres en su libro This new world: the civilization of Latin America. Y, en 1946, Mary Ritter Beard, publicó Woman as force in history: a study in traditions and realities. Otro aporte importante fue el Segundo sexo de Simone de Beauvoir (1949), que influyó de manera relevante y constituye el ensayo feminista más importante del siglo 21. Para Beauvoir, la historia de las

${ }^{1}$ Marc Bloch fue fusilado por los nazis el 16 de junio de 1944 en Lyon. Posteriormente su obra fue publicada por Lucien Febvre con un doble título: Apologie pour l'Histoire ou Métier d'historien. 
mujeres y el análisis de la condición femenina requerían de una antropología estructural y de una historia entonces inexistente.

En esa perspectiva, Michael Foucault (1976) situó el análisis de la explotación vinculada hasta entonces al control político y económico, a una red de poder que incluye a la familia, la cultura, el conocimiento y la sexualidad. Mientras que Philippe Ariès y George Duby, plantearon interrogantes respecto de los límites entre lo público y lo privado, la familia y la sexualidad. Fueron también importantes: Out of our past: the forces that shapped modern America, de Carl Degler (1970), y Women in Iberian expansion overseas, 1415-1815, de Charles Boxer (1975).

En 1973, Michelle Perrot, Pauline Schmitt y Fabienne Bock, dictaron el curso: “'Tienen una historia las mujeres?” En 1982 realizaron un coloquio en Toulouse sobre "Investigaciones sobre la mujer y estudios feministas", y en 1983 volvieron al tema con un seminario titulado: "¿Es posible una historia de las mujeres?" (Perrot, 1984). Concluyendo que sí era posible por la importancia que revisten las relaciones entre la historia de las mujeres, y las distintas corrientes de la historiografía, en la construcción de una historia que intente explicar cómo se producen los significados de la diferencia sexual, "a partir del análisis de los procesos discursivos del poder, que son los que organizan y legitiman las diferencias" (Nash, 1994, p. 62).

Así se fue consolidando un campo específico de la historia de las mujeres gracias al esfuerzo académico de Gerda Lerner, Natalie Zemon Davies, Mary Hartman, Lois Banner, Renata Bridenthal, Claudia Koonz, Sheila Rowbotham, Judith Bennet y Nora Nash, entre otras. Gerda Lerner analizó la formación del patriarcado y el papel de las mujeres en la prolongación de su subordinación; para Natalie Zamon Davis, el objetivo de la historia de las mujeres es descubrir los roles sexuales y el simbolismo sexual en distintas sociedades y periodos con el fin de conocer su significado y cómo funcionaban para mantener el orden ó impulsar el cambio. Mary Hartman y Lois Banner publicaron: Clios consciousness raised: new perspectivas on the history of women (1976); Renate Bridenthal, y Claudia Koonz, Becoming Visible: women in European history (1977); Sheila Rowbotham, Conciencia de mujer en un mundo masculino (1977); Judith Bennet, Feminism and suffrage: the emergence of an independent women's movement in America 1848-1869 (1978).

\section{Las mujeres en el discurso histórico de América Latina}

La intensa movilización social y política en favor de los derechos civiles, la autodeterminación de los pueblos y la independencia política y económica en la década de 1960, posibilitó el cambio del discurso de la historiografía 
en América Latina. En este proceso la historia social cambió su orientación dirigida al estudio del espacio público hacia el espacio privado, y hacia una aproximación a los grupos marginales o carentes de poder, entre los que se encuentran las mujeres. El fin de una historia excluyente en términos de clases, etnias y género, significó el punto de partida para que las mujeres conquistaran su derecho a una historia en la que "dejaron de solo víctimas para convertirse en protagonistas" (Chartier, 2000).

Así mismo, la introducción del género como categoría planteó la necesidad de deconstruir categorías absolutas, identificar la opresión femenina (Lavrin, 1985), y poner "al descubierto los espacios femeninos, los modos de vida particulares de las mujeres, las prácticas culturales que les pertenecían a ellas y no a los hombres". ${ }^{2}$ Fue entonces posible conocer que la pertenencia a uno u otro sexo configuran diferentes actitudes, creencias y códigos en una sociedad determinada. Por ello, los estudios de la mujer y de la historia de las mujeres, no pueden reducir al sexo como sinónimo de sexualidad porque las diferencias físicas tienden a legitimar las relaciones de poder existentes, $\mathrm{y}$ el sexismo al igual que el racismo, le niega al otro "el derecho a ser diferente sin que se le castigue por ello. En otras palabras, se discrimina a aquellos que real o presumiblemente viven, deben vivir, o quieren vivir de un modo distinto al del grupo que dicta las normas y los valores culturales" (Fargue, 1991, p. 64).

En la década de 1980, el interés por "ver" a las mujeres en la historia y el reconocimiento de un campo histórico femenino cobró impulso en América Latina. Se creó el Seminario de la Participación Social de la Mujer en la Historia Contemporánea de México 1930-1964; en 1985, Asunción Lavrin publicó Las mujeres latinoamericanas. Perspectivas históricas (1985), obra que marcó una etapa en los estudios de la historiografía de las mujeres. También en 1985, apareció en el Perú la primera edición del libro de Sara Beatriz Guardia: Mujeres peruanas: el otro lado de la historia ${ }^{3}$ que actualmente está en la quinta edición. Poco después, en 1986, se impartió el primer curso de Historia social de la mujer en México en la Universidad Nacional Autónoma de México. De este período data la obra en cuatro volúmenes, Historia de las mujeres en México. En Uruguay, Silvia Rodríguez Villamil, publicó en 1992 su libro, Mujeres uruguayas a fines del siglo XIX: ¿Cómo hacer su historia?

\footnotetext{
${ }^{2}$ Morant (1995, p. 38) coincide con Hobsbawn cuando sostiene que no es posible trabajar solo con el sexo oprimido, "del mismo modo que un historiador de las clases sociales no puede centrarse por entero en los campesinos".

${ }^{3}$ Primer intento de analizar y estudiar la historia del Perú de manera integral desde una perspectiva de género.
} 
En 1997, se realizó en Lima el primer simposio internacional la mujer en la historia de América Latina, organizado por una comisión presidida por Sara Beatriz Guardia, e integrada por Pablo Macera, director del Seminario de historia rural andina de la Universidad Nacional Mayor de San Marcos; Roland Forgues, director de Andinica. Universidad de Pau, Francia; Concepción Solana, presidenta del capítulo México de la Asociación mundial de mujeres periodistas y escritoras; Marco Martos, director del Instituto de investigaciones humanísticas de la Universidad Nacional Mayor de San Marcos; y Beatriz Prieto, decana del Colegio de bibliotecólogos del Perú. Fue en ese simposio que se creó el Centro de estudios la mujer en la historia de América Latina (Cemhal), la única organización hasta la fecha en América Latina dedicada al estudio de la historia de las mujeres de la región.

\section{Hacia una historia no eurocéntrica ni patriarcal}

Pero en América Latina, además de una historia patriarcal, enfrentamos otro reto que significa la deconstrucción de una historia eurocéntrica que concibe a Europa como el centro, y "al sistema de valores de la cultura europea como el genuino sistema de valores universales" (Fabelo Corzo, 2007, p. 79). En esa perspectiva, si aceptamos el término "descubrimiento" implica admitir que antes de la conquista española no existió ninguna cultura de acuerdo a la ideología según la cual los pueblos indígenas "eran formados por sociedades sin escritura, atrasadas y primitivas, que podrían evolucionar hasta llegar a la "civilización", atravesando siglos desde el momento inicial de la catequización" (Knapp, 2010, p. 81).

Recién a partir de la década de 1970, la orientación de la historia tuvo un notable giro hacia la historia social: elites, criollos, mestizos, comunidades campesinas, Iglesia; historia agraria (tenencia de la tierra, producción, fuerza de trabajo); historia económica (minería, obrajes, gremios, mercado); y la historia demográfica (Cañedo-Arguelles, 1999, p. 89-99). Posteriormente, el desarrollo de la etnohistoria significo un intento sistemático por explicar la originalidad y particularidad del pasado de los pueblos y culturas prehispánicos, y significó un cambio de la visión que se tenía hasta entonces de su organización social, de su economía, y su cultura. Surgió así una historia de las sociedades indígenas como un corpus histórico con su propia lógica, categorías, mecanismos de resistencia y sobrevivencia.

Todo lo cual posibilitó estudiar la condición de las mujeres en las sociedades prehispánicas, el impacto que produjo la conquista, su presencia durante la colonia, en la lucha por la independencia, y en la construcción de los estados nación. Tarea nada fácil respecto de las sociedades andinas, si se tiene 
en cuenta que las principales fuentes de estudio están constituidas por cronistas españoles, en su mayoría sacerdotes, soldados, funcionarios y aventureros, cuya información no solo estuvo orientada a justificar la conquista sino que se hallaba distorsionada por su propia cultura. A la carencia de escritura en los Andes, y el escaso conocimiento que tuvieron los españoles del idioma quechua, lo cual según Garcilaso (1960) era la causa de que el indio entendiese mal lo que el español preguntaba y el español entendiese peor lo que el indio respondía, se añade la visión patriarcal, y una concepción eurocéntrica incapaz de reconocer a otra cultura y a otra sociedad. Por ello, interpretaron y ordenaron el mundo que encontraron según su ideología y las categorías de la Europa del siglo 16.

La mirada de los cronistas tuvo, además, un sesgo de superioridad hacia los indios, a quienes consideraron idólatras, dependientes, e infantiles. Y, así como la tradición intelectual les negó a los pueblos colonizados un lugar en la historia, también lo hizo con las mujeres. Se trata de relatos que narran la conquista y la colonización desde una forma particular de pensar la historia con valores e intereses de una historiografía que no "veía" a las mujeres, y donde la condición de las mujeres indígenas fue interpretada de manera confusa, incorrecta y parcializada. Por ejemplo, la sucesión y las cuestiones relativas a la herencia andina diferían sustancialmente de la concepción hispana. Tampoco el poder fue solo privilegio masculino puesto que las mujeres lo ejercieron también; prueba de ello es la función que cumplieron las coyas, esposas principales del inca, y que algunos repartimientos estuvieron gobernados por mujeres curacas. Esto obedecía a la concepción de dualidad andina, que fue una forma de concebir el mundo conformado por unidades contrarias. "Desde la tierra hasta las aldeas, pasando por el cuerpo humano, los animales y las plantas se entiende que todo tiene dentro principios que luchan entre sí y que, a la vez, se complementan ya que la existencia de cualquiera de ellos es condición para la existencia del otro. Esta multitud de oposiciones binarias consiguen, en conjunto, un equilibrio dentro del cual la vida es posible. El equilibrio no es, por supuesto, permanente" (Lleras Pérez, 2000).

En la estructura social del Virreinato del Perú que comprendía los antiguos territorios del vasto imperio de los Incas que abarcó desde el sur de Colombia, atravesando los actuales territorios de Ecuador, Perú, Bolivia, y el noroeste de Argentina hasta Chile, los conquistadores y sus descendientes conformaron la clase dominante sustentada por tres ejes de poder: la administración pública a cargo del virrey, el cabildo o ayuntamiento integrado por criollos, y la iglesia representada por el episcopado, las órdenes religiosas y el tribunal de la inquisición. Al depender directamente del rey, el clero fue un instrumento 
más en la política de dominación. Al margen de pocas excepciones, apoyó o guardó discreto silencio ante afrentas y ultrajes. La sociedad quedo así dividida en clases que debían mantenerse aisladas para beneficio de la consolidación colonial. Motivo por el cual "se obstruyó toda posibilidad de comunicación y comprensión entre los individuos pertenecientes a los estamentos opuestos" (Tauro, 1993, p. 35).

En el régimen colonial la mujer tuvo que adecuarse a un sistema social complejo y pleno de contradicciones, en cuya base el fraccionamiento de la cultura nativa y el proceso de transculturización tuvieron como marco la opresión y la violencia. En la nueva sociedad, la ideología feudal hispana jugó un rol decisivo en relación a la mujer, sin contar que en el primer período de la conquista los españoles no trajeron a sus mujeres. La emigración de las mujeres españoles hacia América Latina está registrada a partir del siglo 16 de manera muy escueta. La travesía por mar y la epopeya que constituyó para estas mujeres llegar al nuevo mundo, figura en el Archivo general de Indias, en los seis primeros tomos de los libros del "Catálogo de Pasajeros a Indias" de 1509 a 1579, donde se advierte que en esos 70 años llegaron al Virreinato del Perú 7,451, mujeres. Es decir, un promedio aproximado de 106 al año.

Según libros notariales desde fines de la década de 1590 la forma de unión más común durante la colonia fue el concubinato, lo que significó una forma de opresión socioeconómica, étnica y de género, puesto que "en el amancebamiento, la regla general era que el hombres pertenecía siempre a una casta o a una capa social más elevada que de la mujer" (Flores Galindo, 1984). La mujer no sólo fue utilizada sexualmente sino que a los hijos que nacían de estas uniones se les consideraba "ilegítimos", y no podían ingresar a determinados colegios, ni ocupar cargos importantes, ni casarse con quien quisieran.

No es casual que el mayor índice de mortalidad materna fuera ocasionado por prácticas abortivas, no obstante que el aborto estaba prohibido por disposición de Sixto V y Gregorio XIV, y que según el Concilio de Iliberi se negaba a la madre y a sus "cómplices" la absolución en artículo de muerte. El abandono de los recién nacidos también fue un acto "comprensible". Incluso los tratadistas de la época lo llegaron a considerar como un derecho innegable en determinadas circunstancias. José Méndez Lachica, abogado de la Audiencia de Lima, sostenía que "los casados, personas de honor o de extraño fuero podían legítimamente abandonar a sus hijos si los amenazaba la infamia o la pena de muerte" (Macera, 1977, p. 316).

En este contexto, la explotación de los indígenas a través de rígidas formas de subyugación produjo el ingreso más importante del presupuesto español, a la par que jugó un papel relevante en la construcción de las nuevas 
sociedades al convertirse en instrumento de maltratos y atropellos. Al grado que la corona se vio obligada a reglamentar la mita y los obrajes para así detener la acción de los corregidores, crueles ejecutores de un implacable sistema de sujeción.

Según un documento titulado "Presentación de la ciudad del Cusco. Sobre excesos de corregidores y curas", fechado en 1768, y que figura en la Academia de Historia de Madrid, el abuso cometido por los españoles contra los indios era de tal envergadura que el informante hispano no vacila en decirle al rey que: "será preciso apartar la cordura para referirle con claridad que haga ver con cuánta inhumana piedad proceden unos hombres cristianos que, olvidados de su carácter y de toda su razón política, no tendrán semejantes en las menos incultas naciones" (La Rebelión, 1971, p. 4). Mientras que la explotación a la mujer tuvo como signo la violación y el maltrato legitimados por el poder, en relaciones de subyugación a través de las cuales los españoles las convirtieron en sus mancebas, esposas, amantes, sirvientas y prostitutas.

Son numerosos los levantamientos que el sistema de dominación colonial produjo apenas iniciada la conquista cuando en 1538 Manco Inca se sublevó llegando a sitiar el Cusco y Lima, y posteriormente en un período de cuarenta años de resistencia en Vilcabamba. Pero es a partir de la segunda mitad del siglo 18, coincidiendo con la crisis del Virreinato del Perú debido a las reformas borbónicas, que las protestas se suceden de manera constante. Entre 1723 y 1750 se produjeron diez insurrecciones en los actuales países de Chile, Paraguay, Bolivia, Argentina, y Venezuela.

La presencia y participación de las mujeres fue anónima. La historia no registra sus nombres sino a finales del siglo 18 en la rebelión liderada por José Gabriel Condorcanqui Túpac Amaru. Esta significativa presencia con características de liderazgo y heroísmo representadas por Micaela Bastidas tiene origen en la sociedad indígena prehispánica donde las mujeres ocuparon una importante posición, y cuando las circunstancias demandaron, las viudas y hermanas de los jefes fueron "aceptadas como legítimos líderes" (Davies, 2006, p. 134). Queda como testimonio de esta lucha por sus derechos autónomos a la tierra y a ocupar cargos en los gobiernos locales, los juicios e investigaciones de la campaña de extirpación de idolatrías que registraron la voz de las autoridades eclesiásticas españolas y la de los indígenas acusados. En la documentación figuran litigios por títulos de las tierras, así como partidas de matrimonio y bautizo, que permiten reconstruir el intento por defender la tenencia colectiva de la tierra, y la persistencia de los patrones andinos de parentesco: "a lo largo del siglo diecisiete las mujeres continuaron asumiendo el apellido materno, mientras que los hombres tomaban el paterno" (Silverblatt, 1990, p. 172). 
La insurrección de Tupac Amaru estalló el sábado 4 de noviembre de 1780, en un período particularmente importante para la humanidad: cuatro años después de la Declaración de la Independencia de Estados Unidos el 4 julio 1776, y nueve años antes del 14 julio de 1789, cuando el pueblo asaltó la Bastilla en París y se proclamó la Declaración de los derechos del hombre. Se trata de la primera gran insurgencia indígena en América Latina.

Es en el curso de las acciones emprendidas antes de la marcha al Cusco que se registra por primera vez el nombre de Micaela Bastidas que hasta entonces solo figuraba como la esposa del líder rebelde. A diferencia de Tupac Amaru que siempre concitó simpatía y respeto no sólo de la gente más allegada a él, Micaela Bastidas fue calificada de cruel y odiada por los españoles. En varios documentos se refieren a ella con hostilidad asegurando que tenía un carácter más intrépido que el marido, y que en su ausencia dirigía ella misma las expediciones a caballo para reclutar gente y armas, dando órdenes con rara intrepidez y autorizando los edictos con su firma.

Durante los cinco meses que duró el enfrentamiento armado, Micaela Bastidas participó en el combate, y aseguró el suministro de armas y alimentos. La importancia de su presencia en la insurrección queda demostrada en la sentencia:

Por complicidad en la Rebelión premeditada y ejecutada por Tupac Amaru, auxiliándolo en cuanto ha podido, dando las órdenes más vigorosas y fuertes para juntar gente, $[. .$.$] invadiendo las provincias$ para sujetarlas a su obediencia, condenando al que no obedecía las órdenes suyas o de su marido, [...] esforzando y animando a los indios al levantamiento (Antología, 1972, p. 736).

Fue condenada a muerte y ejecutada el 18 de mayo de 1781. Según el visitador José Antonio de Areche, la ejecución de Micaela Bastidas debía ir acompañada "con algunas cualidades y circunstancias que causen terror y espanto al público; para que a vista de espectáculo, se contengan los demás, y sirva de ejemplo y escarmiento" (Bonilla, 1971, p. 175). La ejecución como espectáculo de terror, la "masculinización de su persona percibida en los edictos redactados contra Micaela y en los testimonios legales en torno a su juicio recalcaban la idea de que no merecía ser tratada como una mujer" (Meléndez, 2003, p. 767-769).

Antes de ejecutarla le cortaron la lengua, y como tenía el cuello muy delgado y el torno del garrote no podía ahogarla, los verdugos dándole patadas en el estómago y pechos, la acabaron de matar. Después le cortaron la cabeza, desprendieron sus dos brazos, y sus piernas que fueron enviadas a diferentes ciudades del país, el resto del cuerpo fue quemado. 
La exclusión de género y etnia está pues en la base y en el génesis del sistema de organización social y económica que impusieron los españoles en América Latina a partir del siglo 16. Sin embargo, la exclusión como ideología no cambió con la independencia al culminar el dominio colonial. No aseguró el fin de las guerras civiles ni las tensiones sociales y étnicas, la concentración del poder por los criollos en gobiernos débiles, dominados por el caudillismo, donde los indios y los negros no tuvieron derechos ni ciudadanía.

Tampoco las mujeres. Las primeras constituciones políticas de nuestras naciones estipularon como requisitos para ser ciudadanos: Ser casados, o mayores de veinticinco años, saber leer y escribir. Tener empleo o profesar alguna ciencia o arte. Las nacientes repúblicas legitimaron así un sistema de estratificación social y de exclusión puesto que las mujeres no tenían acceso a la educación y menos a una profesión o empleo.

Exclusión que significa discriminación y pobreza. La existencia de personas o grupos que no pueden acceder a distintos ámbitos de la sociedad, y por consiguiente se trata de desigualdad, y ruptura interna del sistema social. Una forma de violencia estructural, asentada en el régimen colonial y que adquirió legitimidad con la construcción de los estados nación y de nuestras propias identidades. La constante en todo el proceso de independencia de América Latina es la exclusión de género y etnia; los excluidos de la libertad son las mujeres, los indios, los negros (Barros, 1996, p. 89-113). Todo lo cual nos remite a los derechos sociales y a replantear el concepto de ciudadanía en el contexto de sociedades multiétnicas y multiculturales. Problemática que se ubica en el proceso constitutivo de nuestros países con modelos de ciudadanía excluyentes.

\section{La historia de las mujeres: otro derecho por conquistar}

La reconstrucción del pasado femenino supone pues un cambio de paradigma, reformular las categorías del análisis histórico, y por lo tanto rescribir la historia desde una alternativa contestataria con nuevos modelos interpretativos. En buena cuenta, asumir la historia social desde una perspectiva que considere que las relaciones entre los sexos son construcciones sociales, que la dominación masculina es una expresión de la desigualdad de estas relaciones, y en consecuencia producto de las contradicciones inherentes a toda formación social. La construcción de esta historia debe estar centrada en la forma cómo se han percibido y vivido las diferencias sexuales, y en el análisis de una dominación que ha generado distintos grados de sumisión en relaciones de interdependencia con grados insólitos de complicidad de las mismas mujeres (López Chirico, 1992, p. 21). 
La nueva historiografía significa una nueva valoración de las experiencias femeninas mediante una nueva forma de abordar la historia, la revisión de modelos que han impregnado a todos los grupos sociales, y los factores diferenciales que afectan a las mujeres.

Para Jacques Derrida, hay que reemplazar la lógica tradicional practicada en las ciencias sociales por una nueva manera femenina de abordar el pensamiento crítico, siguiendo como señala Scott, una lógica de investigación diferente a la aplicada en la historiografía tradicional. Es decir, rescribir la historia desde una perspectiva femenina, plantear nuevas formas de interpretación, y revisar conceptos y métodos existentes con el objetivo de convertir a las mujeres en sujetos de la historia, reconstruir sus vidas en toda su diversidad y complejidad, mostrando cómo actuaron y reaccionaron en circunstancias impuestas, inventariar las fuentes con las que contamos, y dar un sentido diferente al tiempo histórico, subrayando lo que fue importante en sus vidas (Perotin-Dumon, 2000).

La nueva historiografía significa una nueva valoración de las experiencias femeninas mediante una nueva forma de abordar la historia, la revisión de modelos que han impregnado a todos los grupos sociales, y los factores diferenciales que afectan a las mujeres. Por lo mismo, la construcción de esta historia no puede estar centrada en el eje sujeción - transgresión, sino en el uso que se ha hecho de las diferencias sexuales a través de la historia. Y del análisis de una dominación que abarca miles de años, que ha trascendido a las épocas y a los modos de producción.

Para ilustrar la importancia que tiene la historia de las mujeres, Gerda Lerner demuestra a través de una imagen interesante donde esta el quid de la cuestión. Pensemos, dice, que hombres y mujeres viven en un escenario en el que interpretan el papel, de igual importancia, que les ha tocado. La obra no puede proseguir sin ambas clases de intérpretes. Ninguna contribuye más o menos al todo; ninguna es secundaria o se puede prescindir de ella. Pero la escena ha sido concebida y definida por los hombres. Ellos han escrito la obra, han dirigido el espectáculo, e interpretado el significado de la acción. Se han quedado con las partes más interesantes, las más heroicas, y han dado a las mujeres los papeles secundarios (Lerner, 1990, p. 30).

¿Qué sucede entonces? Cuando las mujeres se dan cuenta de esto reclaman y logran que se las considere en papeles de igual importancia, aunque tengan que pasar por el examen de calificación de los hombres que obviamente eligen a las más dóciles y a las que mejor se adecuan al trabajo que ellos determinan, en tanto castigan con la exclusión a las que se arrogan el derecho de representar su propio papel. Ese es el error, lo que las mujeres debemos 
hacer es escribir también el argumento, intervenir en el escenario de la obra, en la interpretación de los papeles importantes, y en aquellos que consideremos útiles.

La historia de las mujeres se presenta así como un elemento transformador de las mismas mujeres, y constituye un paso decisivo para su emancipación. Una nueva historia significa cambiar todo un andamiaje de ideas y creencias, $\mathrm{y}$ transformar las actividades femeninas en experiencias definidas y trascendentes. No es muy difícil imaginar que entonces sus experiencias y vivencias serán valoradas en el curso del desarrollo de la humanidad, la cultura y la civilización.

\section{Referências}

ANTOLOGÍA de la independencia del Perú. Lima: Publicaciones de la Comisión Nacional del Sesquicentenario de la Independencia del Perú, 1972.

ARIES, Philippe; DUBY, George. Histoire de la vie privée. Paris: Éditions du Seuil, 1999.

BARROS, Paula. Exclusión social y pobreza: implicancias de un nuevo enfoque. Santiago: OIT, 1996.

BEARD, Mary Ritter. America through women's eyes. Connectictut: Greenwood Press, 1969.

BEARD, Mary Ritter. Women as a force in history: a study in traditions and realities. New York: Macmillan, 1946.

BONILLA, José. La revolución de Túpac Amaru. Lima: Ediciones Nuevo Mundo, 1971.

CAÑEDO-ARGUELLES, Teresa. La historia de América ante los nuevos retos. In: Simposio Metodología docente de la historia de América. Pamplona: Asociación Española de Americanistas, Editores Ronald Escobero et al., 1999. p. 89-99.

CHARTIER, Roger. La historia no terminó. El Clarín, Buenos Aires, 28 de agosto del 2000.

DAVIES, Catherine; BREWSTER, Claire; OWEN, Hilary. South American independence: gender, politics, text. London: Liverpool University Press, 2006 $<$ dx.doi.org/10.5949/UPO9781846314117>.

DUBY, Georges; PERROT, Michelle. Histoire des femmes-L'Antiquité. Paris: Plon, 1992.

FABELO CORZO, José Ramón. La ruptura cosmovisiva de 1492 y el nacimiento del discurso eurocéntrico. Graffylia - Revista de la Facultad de Filosofía, v. 7, p. 79-86, 2007.

FARGE, Arlette. La historia de las mujeres: cultura y poder de las mujeres - ensayo de historiografía. Historia Social, v. 9, p. 79-101, 1991.

FOUCAULT, Michael. La volonté de savoir. Paris: Gallimard, 1976. 
FOUCAULT, Michael. Nietzsche, la genealogía, la historia. Valencia: Ediciones Pre-Textos, 1977.

FLORES GALINDO, Alberto. Aristocracia y plebe. Lima: Mosca Azul Editores, 1984.

GARCILASO DE LA VEGA, Inca. Primera parte de los comentarios reales de los incas. Madrid: Biblioteca de Autores Españoles, 1960.

GUARDIA, Sara Beatriz. Mujeres peruanas: el otro lado de la historia. 5. ed. Lima: Cemhal, 2013.

KNAPP, Cássio. Política educacional para a educação escolar indígena. In: Losandro Tedeschi; Antonio Dari Ramos (orgs.). Temas sobre gênero e interculturalidade. Dourados: Cadernos Acadêmicos da UFGD, 2010.

LAVRÍN, Asunción. Las mujeres latinoamericanas: perspectivas históricas. México: Fondo de Cultura Económica, 1985.

LA REBELIÓN de Tupac Amaru. Antecedentes. Colección Documental de la Independencia del Perú. Lima: Comisión Nacional del Sesquicentenario de la Independencia del Perú. Tomo II, Volumen I, 1971.

LERNER, Gerda. La creación del patriarcado. Barcelona: Editorial Crítica, 1990.

LÓPEZ Chirico, Selva. Comentario. In: Silvia Rodriguez Villamil. Mujeres e historia en el Uruguay. Montevideo: Grecmu, 1992.

LLERAS PÉREZ, Roberto. La geografía del género en las figuras votivas de la Cordillera Oriental. Boletín Museo del Oro, n. 47, 2000.

MACERA, Pablo. Sexo y Coloniaje. Trabajos de Historia. Lima: Instituto Nacional de Cultura, 1977. Tomo III, p. 297-346.

MELÉNDEZ, Mariselle. La ejecución como espectáculo público: Micaela Bastidas y la insurrección de Tupac Amaru, 1780-81. In: Carmen Ruiz Barrionuevo. La literatura iberoamericana en el 2000: balances, perspectivas y prospectivas. Universidad de Salamanca, 2003.

MORANT, Isabel. El sexo de la historia. Ayer, v. 17, p. 38, 1995.

MORENO SARDÀ, Amparo. El arquetipo viril protagonista de la historia: ejercicios de lectura_no-androcéntrica. Barcelona: LaSal, edicions de les dones, 1986.

NASH, Mary. Experiencia y aprendizaje: la formación histórica de los feminismos en España. Historia Social, v. 20, p. 151-172, 1994.

NOGAL FERNÁNDEZ, Rocío. Españolas en la arena pública (1758-1808). Buenos Aires: Miño y Dávila, Editores, 2006.

PEROTIN-DUMON, Anne. El género en la historia. Santiago de Chile: University of London, Institute of Latin American Studies, 2000.

PERROT, Michelle. Les femmes ou les silences de l'Histoire. París: Flammarion, 1999.

PERROT, Michelle. Escribir la historia de las mujeres: una experiencia francesa. Ayer v. 15, Barcelona, 1995. 
PERROT, Michelle. Os excluidos da história: operários, mulheres e prisioneiros. Rio de Janeiro: Paz e Terra, 1988.

PERROT, Michelle. Une histoire des femmes est-elle possible? Paris: Rivages, 1984.

RODRÍGUEZ VILLAMIL, Silvia. Mujeres uruguayas a fines del siglo XIX: ¿Cómo hacer su historia? Boletín Americanista, Universidad de Barcelona, v. 33, 19921993.

SCHURZ, William L. This new world: the civilization of Latin America. Nueva York: E. P. Dutton, 1945.

SILVERBLATT, Irene. Luna, sol y brujas: género y clases en los Andes prehispánicos y coloniales. Cusco: Centro de Estudios Regionales Andinos Bartolomé de las Casas, 1990.

SCOTT, Joan W. El problema de la invisibilidad. Género e historia. México: Instituto Mora - Universidad Autónoma Metropolitana, 1992.

SPIVAK, Gayatri Chakravorty. ¿Puede hablar el subalterno? Buenos Aires: El cuenco de plata, 2011.

TAURO, Alberto. Destrucción de los indios. Lima: Universidad Nacional Mayor de San Marcos, 1993.

Recebido em: 8 dez. 2014

Aprovado em: 30 set. 2015

Autora correspondente:

Sara Beatriz Guardia

Malecón Castilla 106

Lima 04 - Perú 